\title{
Numerical Prediction of Flow and Heat Transfer in a Two-pass Square Channel with $90^{\circ}$ Ribs
}

\author{
YONG-JUN JANG ${ }^{\mathrm{a}, *}$, HAMN-CHING CHEN ${ }^{\mathrm{b}, \dagger}$ and JE-CHIN HAN ${ }^{\mathrm{a}, \ddagger}$ \\ ${ }^{\mathrm{a}}$ Turbine Heat Transfer Laboratory, Department of Mechanical Engineering, ${ }^{\mathrm{b}}$ Ocean Engineering Program, \\ Department of Civil Engineering, Texas A \& M University, College Station, TX, 77843, USA
}

(Received 5 May 2000; In final form 27 May 2000)

\begin{abstract}
Numerical predictions of three-dimensional flow and heat transfer are presented for a two-pass square channel with $90^{\circ}$ parallel ribs. Square sectioned ribs were employed along the one side surface. The rib height-to-hydraulic diameter ratio $\left(e / D_{h}\right)$ is 0.125 and the rib pitch-to-height ratio $(P / e)$ is 10 . The computation results were compared with the experimental data of Ekkad and Han (1997) at a Reynolds number $(R e)$ of 30,000.

A multi-block numerical method was used with a chimera domain decomposition technique. The finite analytic method solved the Reynolds-Averaged Navier-Stokes equation in conjunction with a near-wall second-order Reynolds stress (secondmoment) closure model, and a two-layer $k-\varepsilon$ isotropic eddy viscosity model. Comparing the second-moment and two-layer calculations with the experimental data clearly demonstrated that the rib turbulators and the $180^{\circ}$ sharp turn of the channel produced strong non-isotropic turbulence and heat fluxes, which significantly affected the flow fields and heat transfer coefficients. The near-wall second-moment closure model provides an improved heat transfer prediction in comparison with the $k-\varepsilon$ model.
\end{abstract}

Keywords: Second moment closure; Chimera method; Blade internal cooling; Ribbed channel

\section{INTRODUCTION}

Modern gas turbine blades are designed to operate with high heat loads, as the turbine inlet temperature increases, in gas turbine engines. To maintain acceptable blade life, sophisticated cooling techniques such as film cooling and convective internal cooling are essential. For the internal cooling of the blades, the heat transfer augmentation is achieved by using repeated ribs as turbulence promoters. The presence of the ribs leads to a complex flow field such as flow separation and reattachment between the ribs, which produces a high turbulence level that leads to high heat transfer coefficients. Detailed information about the flow and heat transfer characteristics in a

\footnotetext{
* Corresponding author. e-mail: yjj536a@acs.tamu.edu

$\dagger$ e-mail: hcchen@civilmail.tamu.edu

łe-mail: jchan@mengr.tamu.edu
} 
ribbed channel is very important in designing gas turbine engines.

The recent experimental work of Ekkad and Han (1997) provided the most detailed heat transfer coefficient distribution in two-pass square channels with rib turbulators using a liquid crystal transient technique. Their study motivated further numerical investigation and so was used as the basis for the verification of the present calculation.

The earlier computational studies on internal coolant passages with ribs have mostly been restricted to two-dimensional flows, like Liou et al. (1993a). In recent years, some researchers have reported three-dimensional studies. Prakash and Zerkle (1995) performed the calculation of flow and heat transfer in a ribbed $\left(90^{\circ}\right)$ rectangular duct (only one rib-pitch was investigated). Turbulence was modeled with the $k-\varepsilon$ model in conjunction with the wall function. They concluded the low Reynolds number model was necessary to get better results and Reynolds stress model was required to capture the anisotropic effects around the ribs. Stephens and Shih (1995) investigated three-dimensional flow and heat transfer in a rectangular duct (one pass) in which one wall was roughened with five equally-spaced $90^{\circ}$ square ribs. They used a low-Reynolds number $k-\omega$ turbulence model. Rigby et al. (1997) presented the numerical results for flow and heat transfer in a straight channel (one pass) with $90^{\circ}$ parallel ribs using a $k-\omega$ turbulence model. They compared their data with Ekkad and Han (1997) experimental data. The heat transfer coefficient between the ribs showed good agreement with the experimental data. However, the heat transfer coefficient on the top of the ribs was underestimated by this model. Stephens et al. (1996) investigated flow and heat transfer characteristics in a straight duct (one pass) with inclined, $45^{\circ}$ rounded ribs on two opposite walls using a low-Reynolds number $k-\omega$ turbulence model. Iacovides (1998) calculated the periodic flow and heat transfer through the squarecross-sectioned duct (one pitch was studied) with rib-roughened walls. He employed two turbulence models; a $k-\varepsilon$ with a one-equation model of a $k$ transport across the near-wall region and a lowRe differential stress model (DSM). He concluded that the DSM thermal computations were clearly superior to those of the $k-\varepsilon /$ one-equation model, even though the heat transfer coefficient results were not as close to the experimental data as the flow prediction.

Rigby (1998) presented a numerical prediction of flow and heat transfer in a ribbed $\left(90^{\circ} \mathrm{rib}\right)$ coolant passage with a $180^{\circ}$ turn. The computation was performed using a $k-\omega$ turbulence model. Iacovides and Raisee (1999) explored turbulence modeling issues related to the computation of flow and heat transfer in internal cooling passages of turbine blades with normal ribs $\left(90^{\circ}\right)$. They tested four turbulence models: zonal $k-\varepsilon$, a low-Re $k-\varepsilon$, a zonal differential stress model (DSM), and a lowRe DSM. They found that zonal models underpredict surface heat transfer coefficients because they ignored the effects of transport on the nearwall turbulence scale. The low-Re closures were found to be able to reproduce the correct surface heat transfer coefficients. In their results, they found that the low-Re DSM model reproduced the turbulence field more faithfully than the $k-\varepsilon$ model.

There are three main reasons why extensive numerical studies of the flow and heat transfer in ribbed ducts with sharp $180^{\circ}$ turn have not been possible to date. First, detailed experimental data around/on the ribs were not available. The second, as indicated in Iacovides and Raisee (1999), wall function approach was inappropriate when there were flow separations. Moreover, the simple isotropic eddy viscosity model could not capture the physically reasonable flow and heat transfer behavior induced by the anisotropic characteristic of turbulent flow around the ribs and the $180^{\circ}$ sharp turn region. Third, for flows within a strong curvature, which causes flow separation, it has been necessary to employ high order schemes for the discretization of convective transport.

However, recent computational work by Chen et al. (2000) demonstrated the superiority of the second-order Reynolds stress closure over simpler $k-\varepsilon$ isotropic eddy viscosity models using the finite 
analytic method in rotating two-pass square channels with smooth walls. Encouraged by this successful result, this model has been used to predict the flow and heat transfer in ribbed channels. This paper presents the prediction of flow and heat transfer characteristic in a two-pass square channel, with $90^{\circ}$ parallel ribs, that was experimentally investigated by Ekkad and Han (1997). The computation was performed by a twolayer $k-\varepsilon$ eddy viscosity model and also a near-wall second-moment turbulence closure model in order to assess its effectiveness. The governing equations for this model were described in detail in Chen et al. (2000) and will not be repeated here. For completeness, we will briefly summarize the numerical method in the following section.

\section{CHIMERA RANS METHOD}

In the present study, the chimera RANS method of Chen (1995a, 1995b) and Chen, Jang and Han (2000) was employed for the calculation of fluid flow and heat transfer in stationary ribbed channels. The present method solved the mean flow and turbulence quantities in arbitrary combinations of embedded, overlapped, or matched grids using a chimera domain decomposition approach. In this approach, the solution domain was first decomposed into a number of smaller blocks to facilitate efficient adaptation of different block geometries, flow solvers, and boundary conditions for calculations involving complex configurations and flow conditions. Within each computational block, the finite-analytic numerical method of Chen and Chen (1998) and Chen, Patel and Ju (1990) was employed to solve the unsteady RANS equations on a general curvilinear, bodyfitted coordinate system. The coupling between the pressure and velocity was accomplished using a hybrid PISO/SIMPLER algorithm given by Chen and Patel (1989) and Chen and Korpus (1993). The method satisfied continuity of mass by requiring the contravariant velocities to have a vanishing divergence at each time step. Pressure was solved by using the concept of pseudovelocities and, when combined with the finiteanalytic discretization gives the Poisson equation for pressure. To ensure the proper conservation of mass and momentum between the linking grid blocks, the grid-interface conservation techniques of Hubbard and Chen (1994) and Chen and Chen (1998) were employed to eliminate the unphysical mass source resulting from the interpolation errors between the chimera grid blocks. More detailed descriptions of the chimera RANS method were given in Chen and Chen (1998) and Chen and Liu (1999).

\section{RESULTS AND DISCUSSION}

Calculations were performed for the two-pass square channel with $90^{\circ}$ ribbed wall as tested by Ekkad and Han (1997). Figure 1 shows the geometry and the numerical grids for the one side ribbed channels. The ribbed side wall was denoted as the bottom surface and the opposite side wall was denoted as the top surface. The other two side walls were denoted as the inner and outer surfaces. A total of nine ribs were simulated. Four ribs are in the first passage and another four ribs are in the second passage. There was a $90^{\circ} \mathrm{rib}$ in the turn region. All ribs were normal to the flow direction, at an angle $(\alpha)$ of $90^{\circ}$.

The length of the duct $(L)$ was $8.375 D_{h}$. The length from the inlet to the first rib $\left(L_{1}\right)$ was $3.5 D_{h}$ and the length from the last rib in the first passage to the outer surface in the bend $\left(L_{2}\right)$ was $1.0 D_{h}$. The length from the divider wall tip to the outer surface in the bend $\left(L_{3}\right)$ was $1.0 D_{h}$. The divider wall thickness $(d)$ was $0.25 D_{h}$. The rib height-tohydraulic diameter ratio $\left(e / D_{h}\right)$ was 0.125 and the rib pitch-to-height ratio $(P / e)$ was 10 . The arc length $(S)$ was measured from the middle of the turn. Positive $S$ indicated the second passage and negative $S$ indicated the first passage.

A fully developed turbulent boundary layer profile was used at the inlet of the duct in the present calculations. Since the fully developed 
profiles for mean flow and turbulence quantities were not known analytically, a separate calculation was performed for a straight duct to provide the inlet conditions at the duct entrance. The flow was assumed to be parabolic at the exit of the duct with zero-gradient boundary conditions for mean velocity and all turbulence quantities, while linear extrapolation was used for the pressure field. All walls including the rib surfaces were heated to a constant temperature. The coolant fluid at the entrance of the duct was air, at a uniform temperature, $T_{o}$ (i.e., $\left.\theta=\left(T-T_{o}\right) /\left(T_{w}-T_{o}\right)=0\right)$ and the wall temperature, including the ribs, was kept constant at $T=T_{w}(\theta=1)$. Reynolds number $(R e)$ was fixed at 30,000 .

The Nusselt numbers presented herein were normalized with a smooth tube correlation (Kays and Crawford, 1993) for fully developed, nonrotating, turbulent flow:

$$
N u_{o}=0.0176 R e^{0.8}
$$

The present numerical grid was generated using an interactive gridding code GRIDGEN. It was then reblocked into several interlocked computational blocks to facilitate the implementation of near-wall turbulence models and specification of boundary conditions. To provide adequate resolutions of the viscous sublayer and buffer layer adjacent to a solid surface, the minimum grid spacing in the near-wall region was maintained at $10^{-4}$ of the body length which corresponded to a wall coordinate $y^{+}$of the order of 0.1 . In all calculations, the root-mean-square (rms) and maximum absolute errors for both the mean flow and turbulence quantities were monitored for each computational block to ensure complete convergence of the numerical solutions. A convergence criterion of $10^{-5}$ was used for the maximum rms error in all computational blocks.

A grid-refinement study was performed using four different grid distributions. In the first test, three grid systems $21 \times 21 \times 436,41 \times 41 \times 436$,

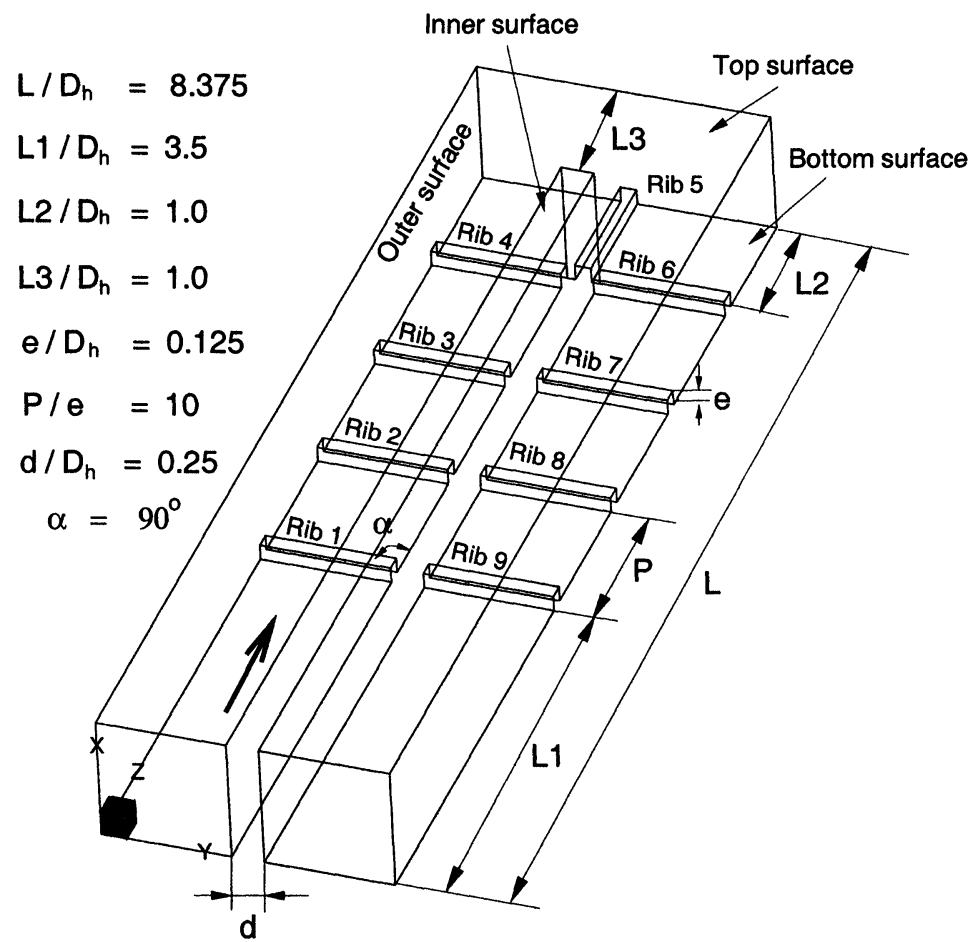

FIGURE 1 Geometry and numerical grids. 


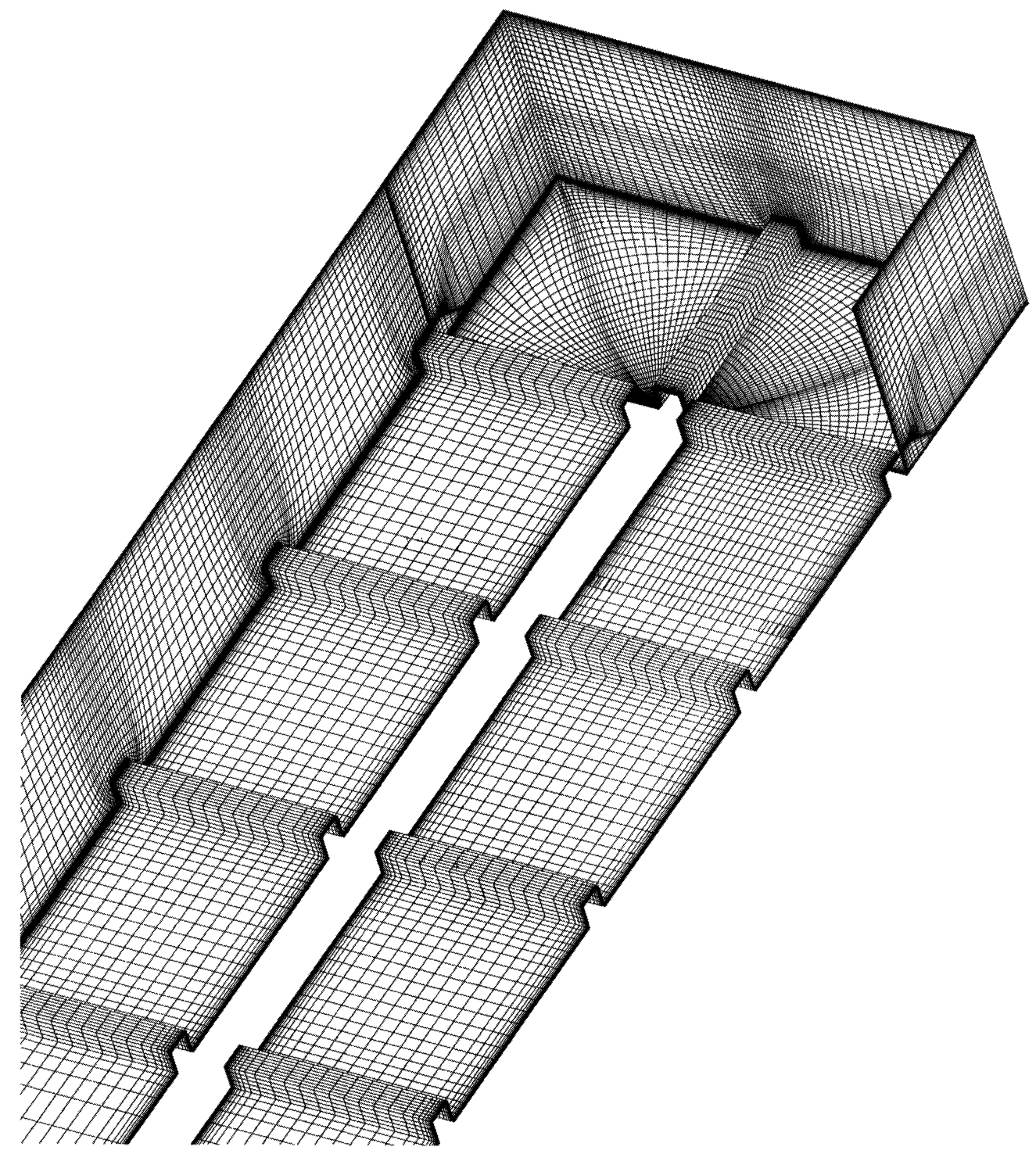

FIGURE 1 (Continued).

$61 \times 61 \times 436(436$ in the streamwise direction and 21,41 and 61 in each of the two cross-stream directions) were evaluated. The maximum improvement in the spanwise averaged Nusselt number ratio was about $40 \%$ when the numerical grid was refined from $21 \times 21 \times 436$ to $41 \times 41 \times 436$. Further grid refinement to $61 \times$ $61 \times 436$ produced only $4 \%$ improvement in the straight regions and $10 \%$ improvement in the bend for the predicted spanwise averaged Nusselt number ratio. In the second test, two grid systems of $41 \times 41 \times 218$ and $41 \times 41 \times 436$ were examined to determine the grid resolution in the axial direction. The Nusselt number ratio was improved by $5 \%$ with $41 \times 41 \times 436$ compared to $41 \times 41 \times 218$. The findings from this investigation showed that the grid system $41 \times 41 \times 280$ for the straight regions and $61 \times 61 \times 125,61 \times 61 \times 31$ 
for the bend and regions between ribs 6 and 7 in second passage, respectively were nearly grid independent. The grid was divided into five computational blocks with a total grid point of approximately $1,060,000$ points.

\section{Mean Velocity and Temperature Fields}

The three-dimensional mean flow and temperature fields for a two-pass square channels with $90^{\circ}$ parallel ribs are presented in this section. For the sake of brevity, we will only present the details of the flow and temperature fields for the secondmoment solutions, since the second-order Reynolds stress model produced more accurate results for all test cases. The two-layer $k-\varepsilon$ results will be discussed only in the comparison of Nusselt number ratio distributions to quantify the effects of Reynolds stress anisotropy.

The velocity vector distributions in the planes midway between the top and bottom surfaces are shown in Figure 2. Unlike the smooth duct case in which flow separation occurred near the divider wall tip in the bend and near the inner surface in the second passage (Jang et al., 2000), separation bubbles did not exist near the divider wall tip in the bend region. Even immediately downstream of the bend, there was no separation bubble. This phenomenon attributed to the presence of ribs in three locations; immediately upstream of the bend, in the middle of the bend and immediately downstream of the bend. Those ribs reduced the centrifugal effect in the bend. Figure 3 shows the streamwise velocity and temperature profiles at both the first and second passages in the planes midway between the inner and outer surfaces. The reversal flow occurred immediately downstream and upstream of the ribs unlike the $60^{\circ}$ angled ribbed duct in which there was no reversal flow immediately upstream of the ribs as indicated by Jang et al. (2000). In the first passage, the reattachment length was about 3.4 times the rib height, which was consistent with the Ekkad and Han (1997) data. After the bend, a strong flow impingement occurred on the bottom surface between ribs 6 and 7, which was due to the bend effect. Temperature fields on the bottom surface were disturbed by the presence of the ribs (Fig. 3(b)). The periodic ribs broke the viscous sublayer and created local wall turbulence due to the flow separation and reattachment between the ribs. This caused thinner thermal boundary layer

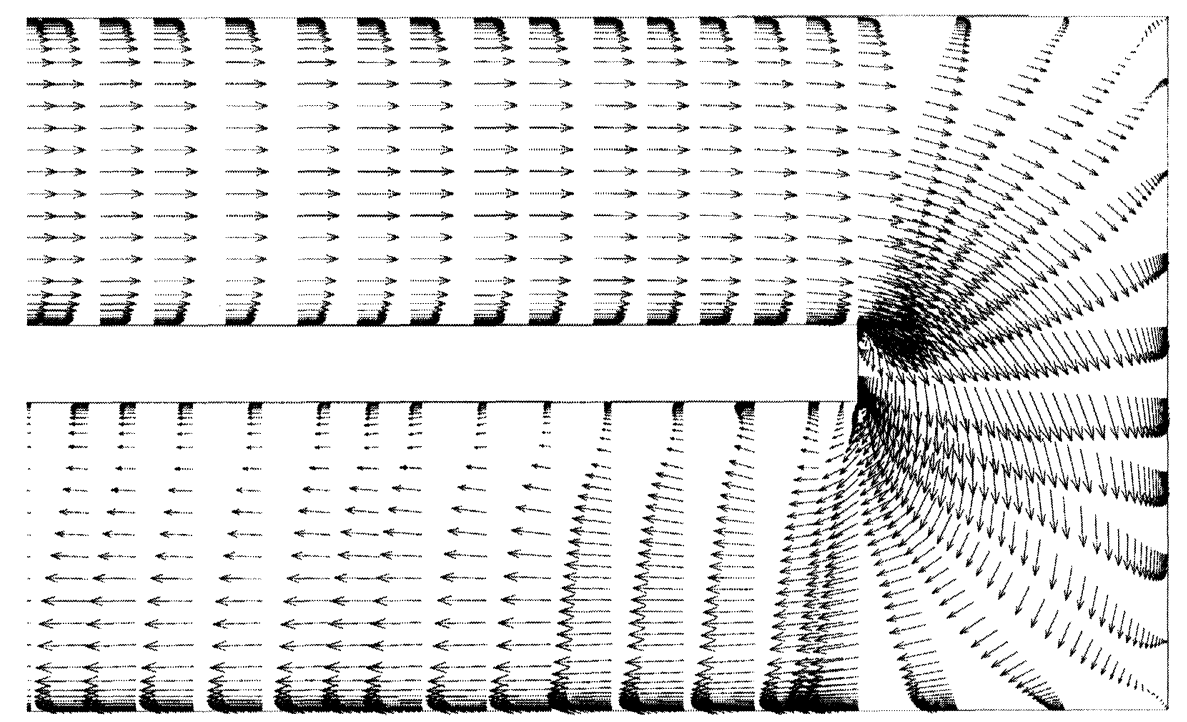

FIGURE 2 Streamwise velocity vector midway between the top and bottom surfaces. 


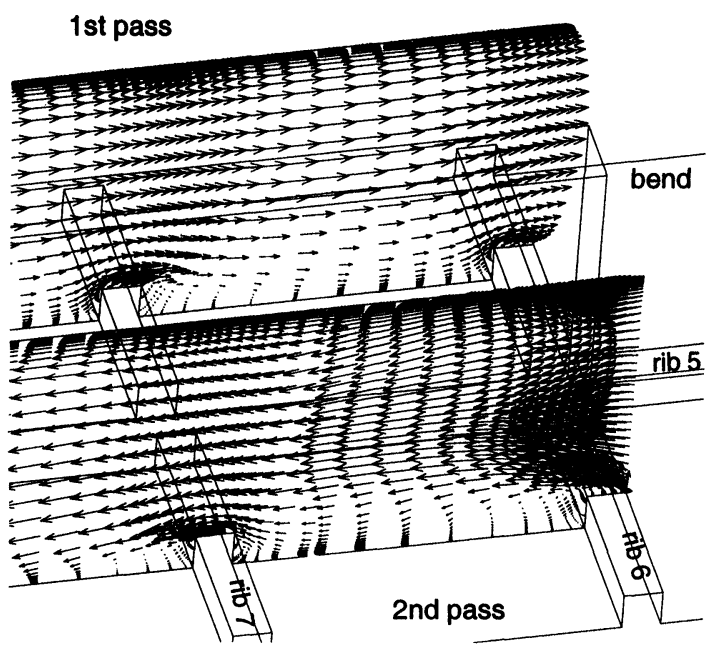

(a)

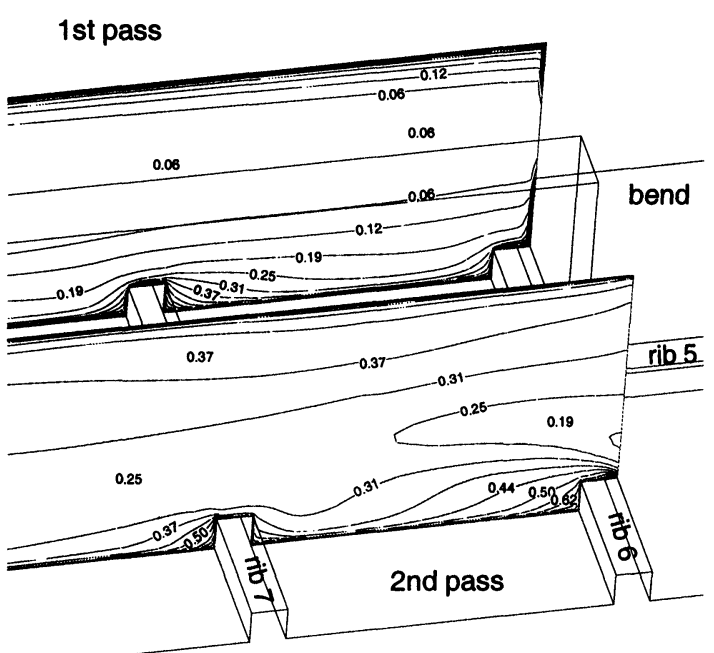

(b)

FIGURE 3 Streamwise velocity and temperature fields midway between the inner and outer surfaces.

on the bottom surface (Fig. 3(b)). After the bend, much higher temperature existed due to the flow mixing by centrifugal force in the bend.

Figure 4 shows the cross-stream velocity vectors and the isothermal contours at selected planes. Section A is on the top of rib 2 and Section B is on the $6.1 \mathrm{rib}$ height of flow development downstream of the rib 2 and Section $C$ is on the midway between ribs 3 and 4 in the first passage. Section $D$ is on the top of rib 5 in the center of the bend Section $\mathrm{E}$ is on the midway between ribs 6 and 7 and Section $F$ is on the top of rib 7 and Section $\mathrm{G}$ is on the midway between ribs 7 and 8 in the second passage. As shown in Figure 4(a)-1, $90^{\circ}$ parallel-ribbed channel produced periodically
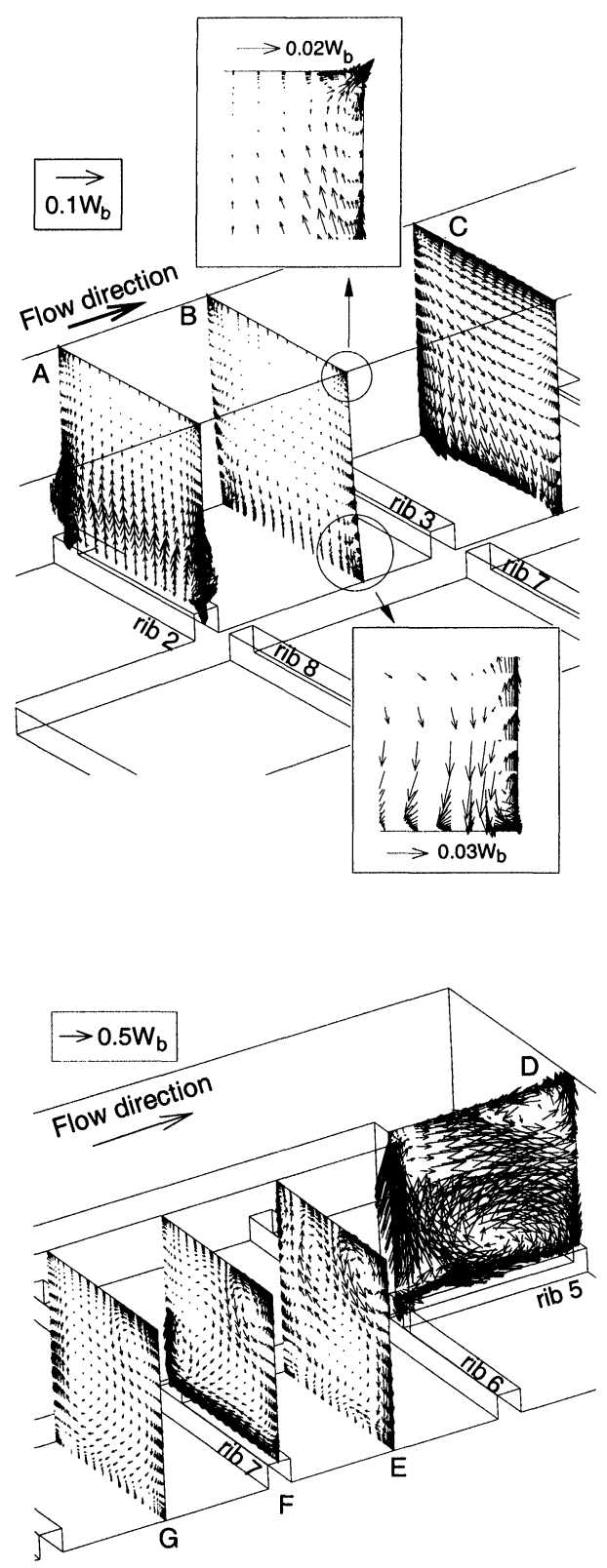

FIGURE 4 Secondary flow and temperature fields $\left(\theta=\left(T-T_{o}\right) /\left(T_{w}-T_{o}\right)\right)$ 


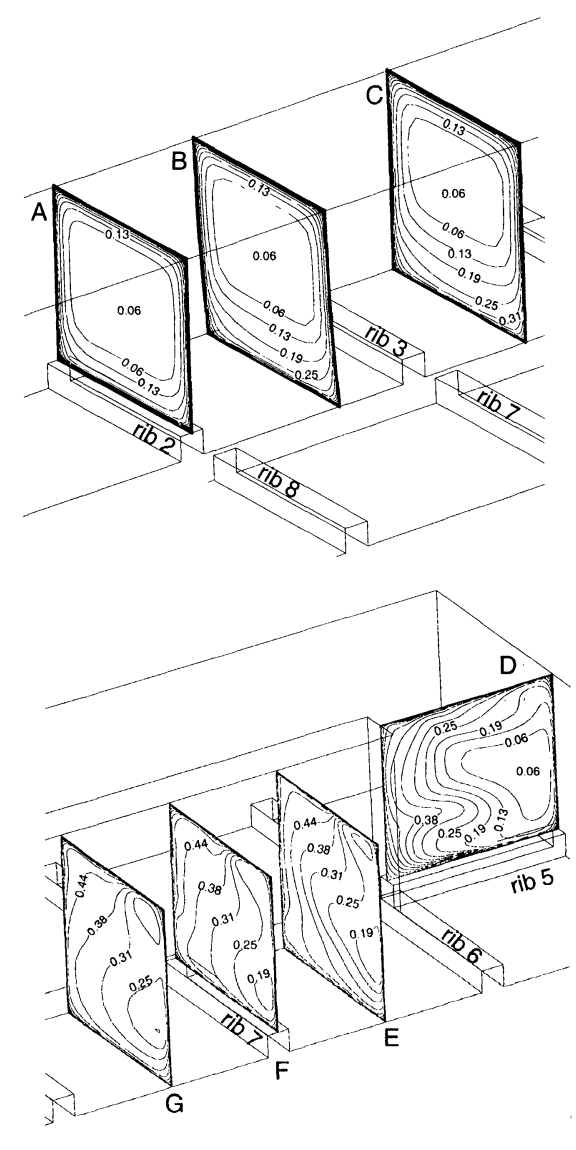

FIGURE 4 (Continued).

up-and-down flow movement and simultaneously generated the small vortex near the bottom surface on the both inner and outer surfaces, which was measured by Liou et al. (1993b) using LDV. On the other hand, secondary flow occurred near the top surfaces (Fig. 4 (a)-1) was not generated by $90^{\circ}$ rib arrangement but by non-isotropic turbulence. The secondary flow pattern in Section $C$ was completely different with Sections A and B due to the bend effect. In the bend, a strong vortex was generated near the top of the rib 5 and smaller one occurred near the top and outer surfaces due to the combined effect of centrifugal-induced vortex in the bend and rib-induced vortex in the upstream (Fig. 4(a)-2). The secondary flow pattern in the second passage is more complicated due to the combined effect of the bend and ribs. In Section E, three vortices were generated and velocity magnitude was very high compared to the one in the first passage. It could be seen that the bend effect persisted farther downstream of the rib 7. The secondary flow structure generated in the first passage transported the cooler fluid from the core toward the top surface. This led to steep temperature gradients on the top surface as shown in Figure 4(b)-1. In the bend, the cooler fluid is pushed toward the outer surface due to the centrifugal force in this region. In the second passage, the secondary flows pushed the cooler fluid toward outer surface and farther downstream, in the Section G, temperature was pretty mixed.

\section{Reynolds Stresses}

The calculated Reynolds stress normal components are presented in Figures 5 and 6. Figure 5 shows the Reynolds stress normal components midway between the inner and outer surfaces in the first and second passages. As the overall characteristics of the Reynolds stresses in the ribbed channel, the ribs produced a high level of

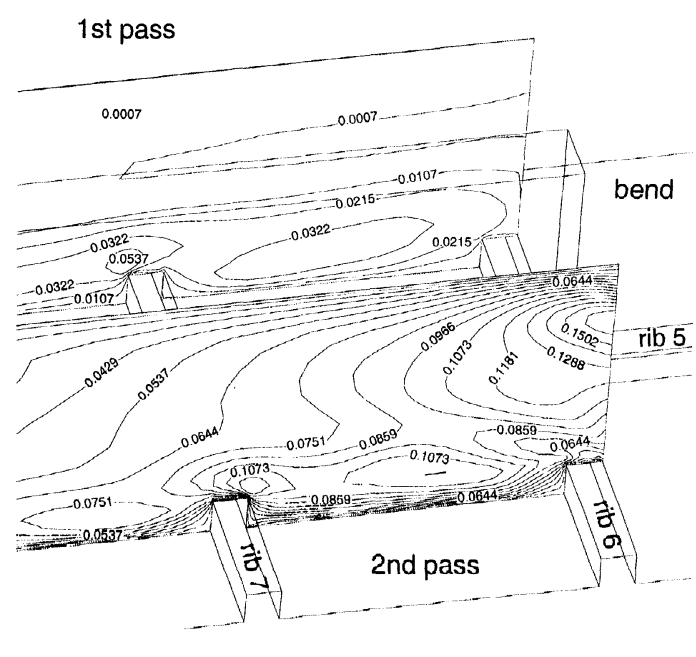

(a) $\overline{u u} / W_{b}^{2}$

FIGURE 5 Reynolds stress components midway between the inner and outer surfaces. 


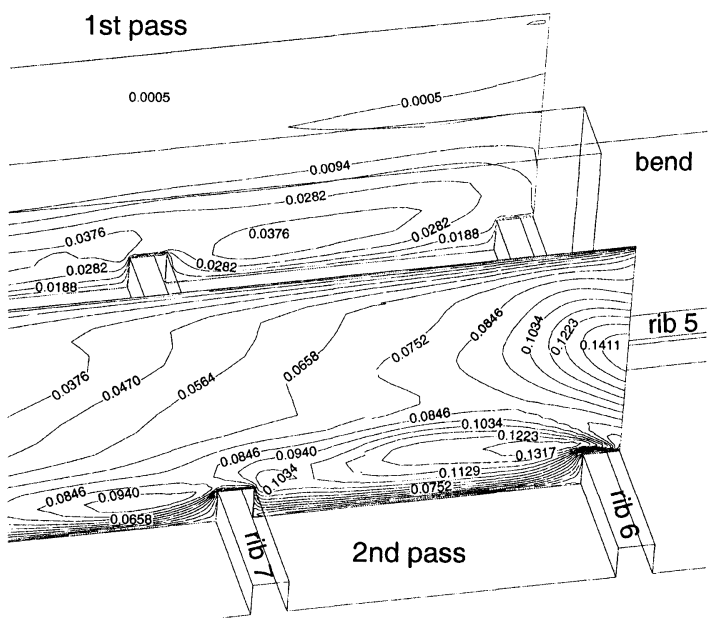

(b) $\overline{v v} / W_{b}^{2}$

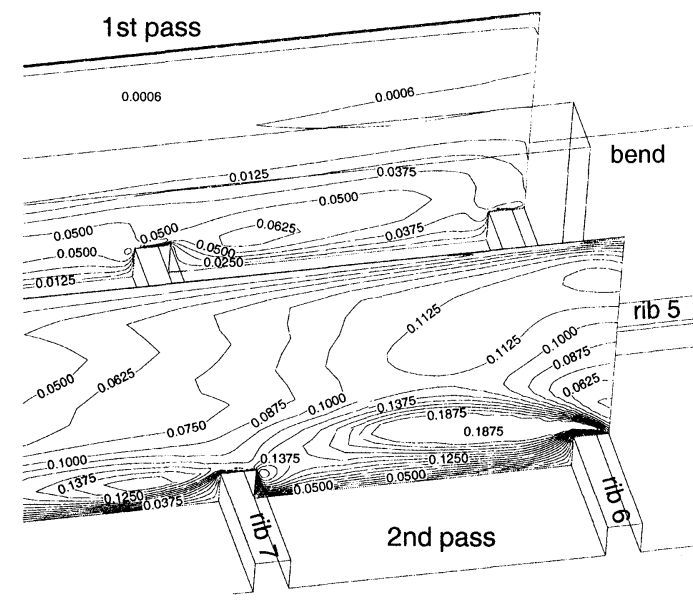

(c) $\overline{w w} / W_{b}^{2}$

FIGURE 5 (Continued).

turbulence. In the first passage, turbulence intensity $\left(\sqrt{\overline{w w}} / W_{b}\right)$ was as high as $25 \%$ immediately downstream of the ribs and diminished gradually toward the smooth top surface, $\left(\sqrt{\overline{w W}} / W_{b} \sim 3 \%\right)$. A high degree of anisotropy $(1.5 \leq \overline{w w} / \overline{u u}$ or $\overline{w w} / \overline{v v} \leq 2$ ) was also observed downstream of each rib. In the second passage, the level of turbulence intensity increased due to the bend effect. $\sqrt{\overline{w w}} / W_{b}, \sqrt{\overline{v v}} / W_{b}$ and $\sqrt{\overline{u u}} / W_{b}$ were about $23 \% \sim 43 \%$ in the region between ribs 6 and 7 . The high turbulence intensity for $\sqrt{\overline{w w}} / W_{b}$ occurred immediately on the top of rib 6 and was transported downstream (Fig. 5(c)). The degree of the anisotropy increased up to 2.9. Farther downstream in the second passage, turbulence intensity level decreased to $16 \% \sim 37 \%$. Figure 6 shows the Reynolds stress components at selected cross sections (A, B, C, D, E, F, G are the same locations as those defined in Fig. 4). In the first passage, high turbulence intensities $(15 \%-25 \%)$ occurred on the top of the ribs and in the regions of reattachment between the ribs. These Reynolds stresses were transported toward the top surface due to the rib-induced secondary flow patterns in the first passage. Thus, the turbulence level was relatively high near the bottom and is low near the

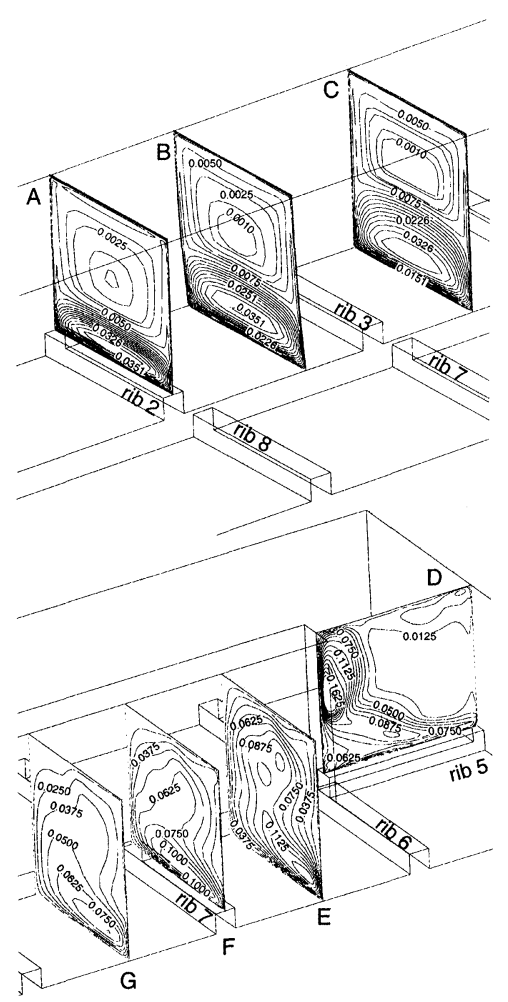

(a) $\overline{u u} / W_{b}^{2}$

FIGURE 6 Reynolds stress components at selected cross section. 


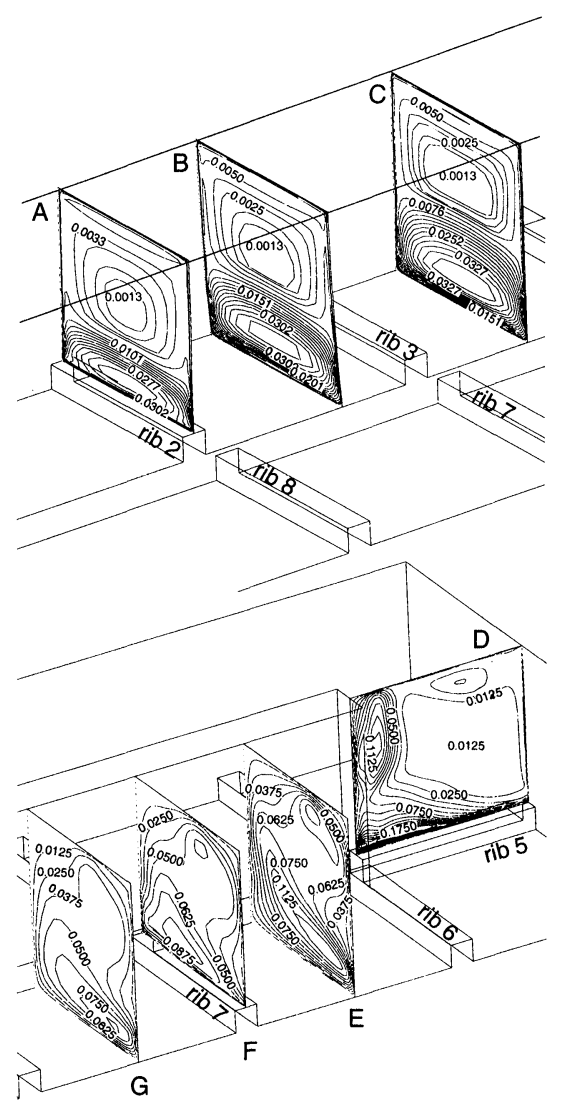

(b) $\overline{v v} / W_{b}^{2}$

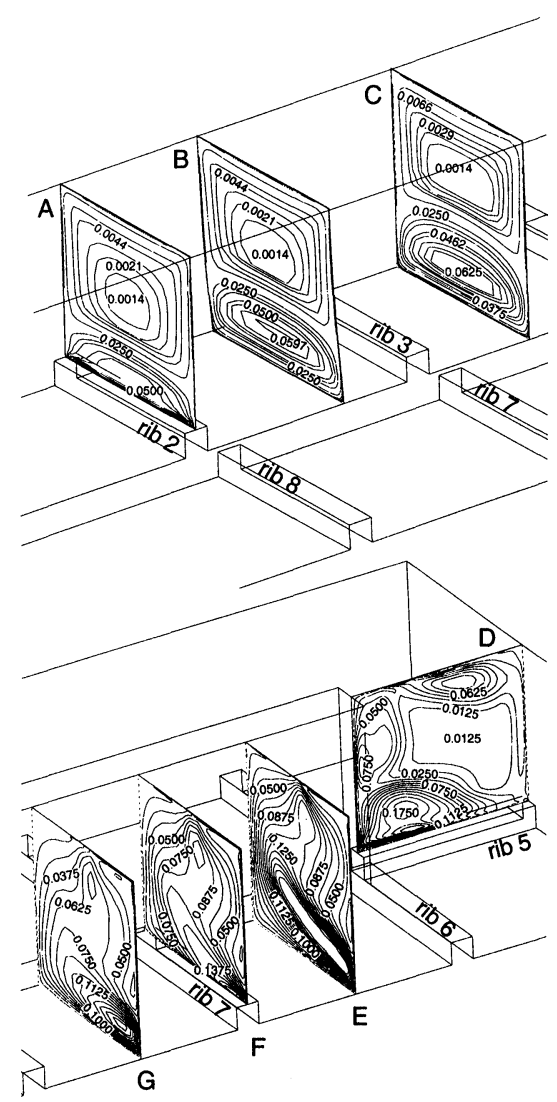

(c) $\overline{w w} / W_{b}^{2}$

FIGURE 6 (Continued).

top surface. In the first passage, the degree of anisotropy was about $1 \leq \overline{w w} / \overline{u u}$ or $\overline{w w} / \overline{v v} \leq 2$. In the bend, turbulence level was still high on the top of rib: where the turbulence intensity was about $27 \% \sim 41 \%$. The secondary flow in the bend transported the high Reynolds stresses toward the inner and top surfaces. The degree of the anisotropy was as high as 2.3. In the second passage, the peak turbulence intensity $(\sim 35 \%)$ occurred around the bottom and outer surfaces in Section E (between ribs 6 and 7). The reason for this is high shear layer in that location, which was caused by the bend effect. Farther downstream, the general turbulence level was down to about $10 \% \sim 30 \%$. The degree of the anisotropy $(\overline{w w} / \overline{u u}$ or $\overline{w w} / \overline{v v})$ in the second passage was about $1.5 \sim 2$.

\section{Surface Heat Transfer}

Figure 7 shows the detailed Nusselt number ratio distributions in a two-pass channel with $90^{\circ}$ parallel ribs. The highest Nusselt number ratios were obtained on the top of ribs in both passages of the channel. Heat transfer distributions between adjacent ribs appeared periodic in the first passage. Nusselt number ratio was high in the middle region between two ribs, and very low immediately before and after the ribs. In the turn region, heat transfer was enhanced greatly due to the combination of the sharp $180^{\circ}$ turn and the $90^{\circ}$ ribs. The presence of the ribs appeared to reduce the effect of centrifugal forces on the secondary flow and caused lesser impingement on the outer surfaces. 
(a) two-layer model $(k-\varepsilon)$

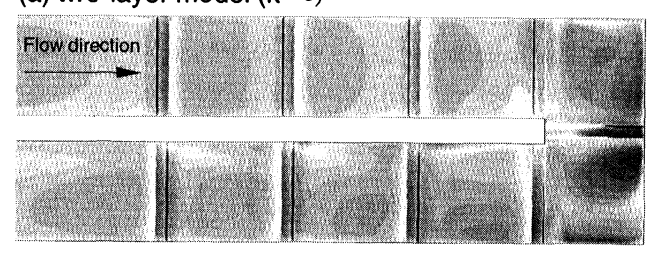

(b) Second-moment model

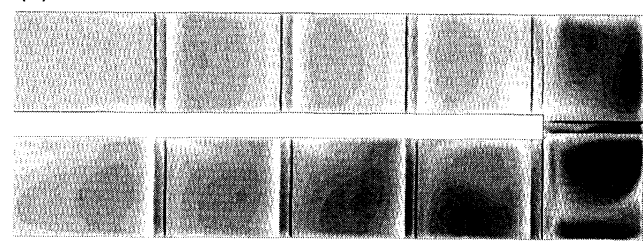

(c) Ekkad and Han (1997)

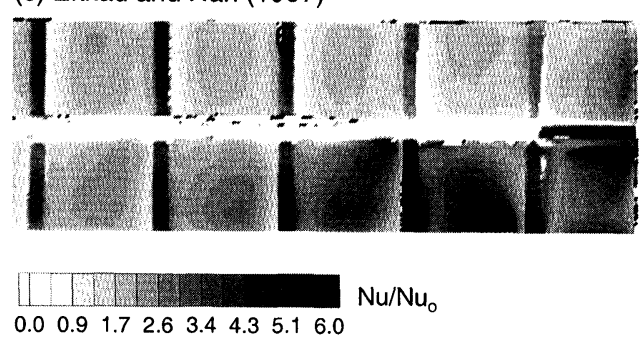

FIGURE 7 Detailed Nusselt number ratio distributions.

Locally high heat transfer region was obtained immediately downstream of the ribs in the turn and second passage. Nusselt number ratios decreased with the reduction in the effect of the turn. Twolayer calculations predicted well the heat transfer patterns in the entire channel. However, it underpredicted the level of Nusselt number ratio in the entire channel. Whereas, second-moment calculation results are in good agreement with the Ekkad and Han (1997) data.

Figure 8 presents an enlarged view of the detailed Nusselt number ratio distributions on all four surfaces around ribs 2, 3 and 4 in the first passage and ribs 6,7 and 8 in the second passage. The highest heat transfer coefficients in the first passage took place on the front side of the rib due to flow impingement (Fig. 8(a)). It could be clearly seen that there was a flow separation and reattachment on the top of the rib (Fig. 8(a)). In the regions between the ribs, the heat transfer was high downstream of each rib because the flow reattachment took place there, so that the thermal boundary layer was thinner (Fig. 8(a)). Due to the rib-induced secondary flow characteristics of this configuration, the heat transfer decreased downstream of the rib to upstream of the next rib in the streamwise direction. Heat transfer on the inner and outer surfaces near the bottom surface was high due to the secondary flow in these regions (see also Fig. 4(a)-1)

In the second passage, the Nusselt number ratio steeply increased on all four surfaces due to the strong flow impingement and reattachment. The Nusselt number ratios decreased as the flow moved downstream. Immediately downstream of the bend, Nusselt number ratio on the top surface was as high as that of the bottom surface.

\section{Spanwise-averaged Heat Transfer Distribution}

The spanwise average Nusselt number ratios on the bottom surface (ribbed surface) for a two-pass square channel with $90^{\circ}$ parallel ribs are shown in Figure 9. Comparisons were made between the calculations and the experimental data of Ekkad and Han (1997). The numerical results obtained from both the second-moment closure model and the two-layer eddy viscosity models were presented to facilitate a detailed assessment of the effect of the Reynolds stress anisotropy. The spanwiseaveraged Nusselt number distributions on the ribbed surface showed the periodic spikes, where the higher ones were caused by the flow impingement on the ribs and the smaller ones were caused by the flow reattachment between the ribs. The Nusselt number ratios were low immediately upstream and downstream of the ribs. The after-turn region had the highest Nusselt number ratios due to the strong effects of both the $180^{\circ}$ sharp turn and the presence of ribs. Farther downstream, the effect of turn reduced.

The predicted Nusselt number ratios by the second-moment closure model were close to the Ekkad and Han (1997) data. As a conclusion from Figures 7 and 9 , the heat transfer prediction by a 
two-layer model had a good trend. However, twolayer model failed to capture the steep increases of the heat transfer between the ribs. On the other hand, the near-wall second-moment solutions were in considerably better agreement with the experimental data. Since both the two-layer and second-moment calculations were performed using the same numerical method and grids, the improved prediction could clearly be attributed to the inclusion of the Reynolds stress anisotropy

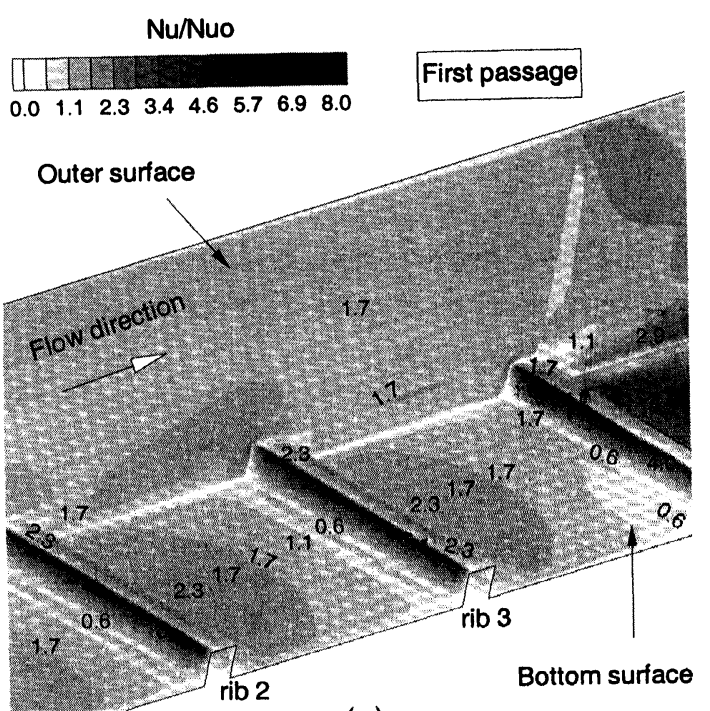

(a)

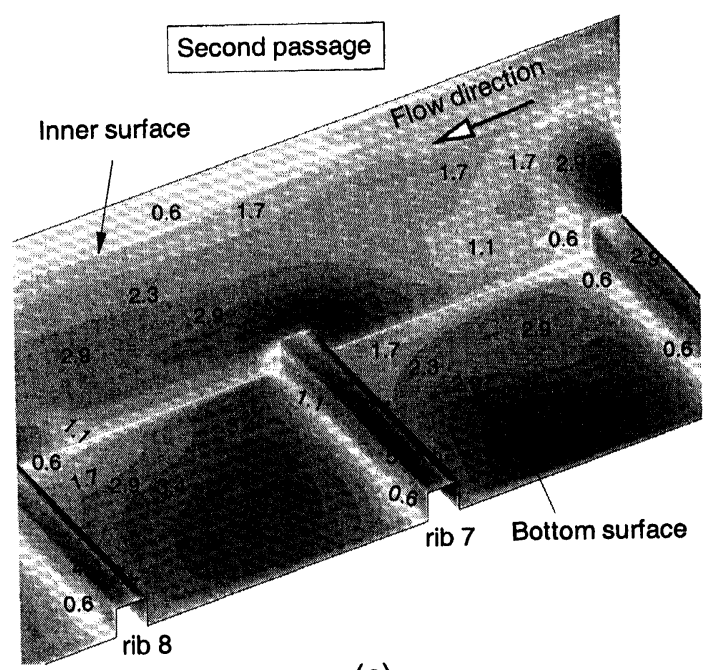

(c)

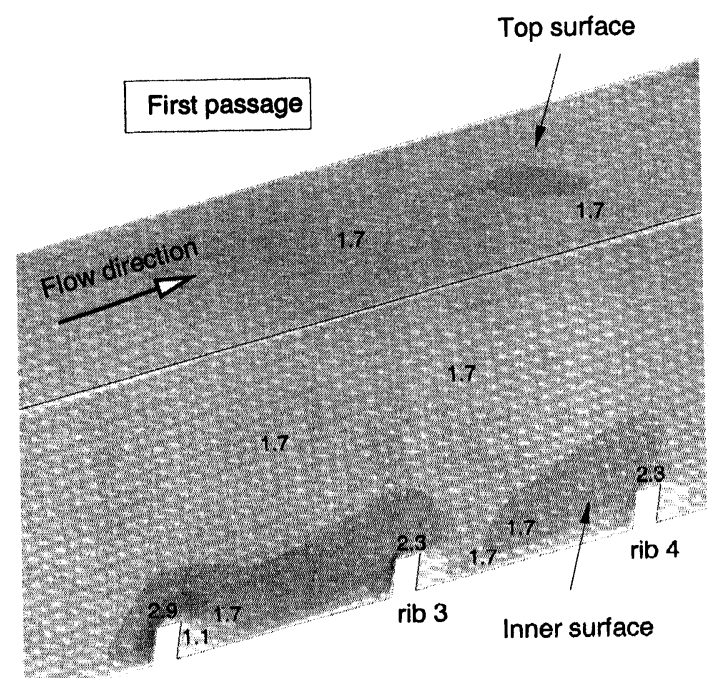

(b)

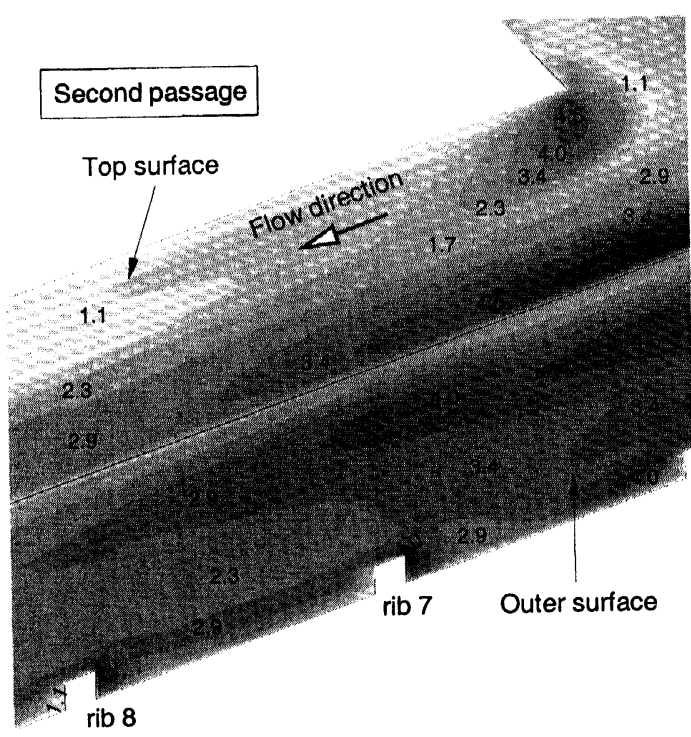

(d)

FIGURE 8 Enlarged view of detailed Nusselt number ratio distribution. 


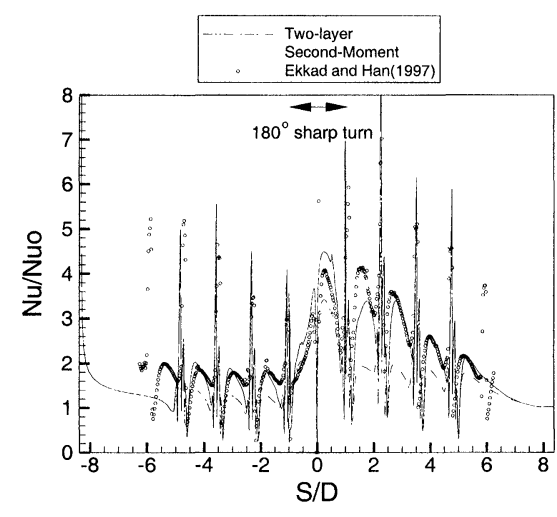

FIGURE 9 Calculated and measured Nusselt number ratios. $(R e=30,000)$.

in the present second-order Reynolds stress closure model.

\section{CONCLUSIONS}

Numerical prediction of flow and heat transfer are presented for a two-pass square channel with a $90^{\circ}$ ribbed wall. The method solved Reynolds-Averaged Navier-Stokes equations in conjunction with a near-wall second-order Reynolds stress closure model for accurate resolution of the turbulent flow and thermal fields produced by normal ribs and $180^{\circ}$ sharp turn. Calculations were also performed using a two-layer isotropic eddy viscosity model to facilitate a detailed assessment of the second-order effects due to the Reynolds stress anisotropy.

The obtained numerical results clearly demonstrate the superiority of the present second-order Reynolds stress closure models over simpler isotropic eddy viscosity models. In general, the second-moment solutions exhibit a significant level of anisotropy in the normal components of a Reynolds stress tensor. For the ribbed two-pass square channels considered here, the normal ribs with a high blockage ratio and a $180^{\circ}$ sharp turn produced strong non-isotropic turbulence that significantly influence the development of momentum and thermal boundary layers along the duct.
Specially, in the bend, the higher level of anisotropy of Reynolds stresses occurred in all of the regions which rendered the isotropic eddy viscosity model failed to predict the complex flow behaviors. Therefore, it is important to employ secondmoment closure models that solve each individual Reynolds stress component directly from their respective transport equations. The present nearwall second-moment closure model accurately predicts the complex three-dimensional flow and heat transfer characteristics resulting from the normal ribs and strong wall curvatures. It provides the reliable numerical predictions, which are in good agreement with the experimental data of Ekkad and Han (1997).

\section{Acknowledgements}

This work was supported by the Texas Higher Education Coordinating Board - Advanced Technology Program under grant number 999903-165. The computations were performed on the Cray J90 at the Texas A \& M Supercomputer Center under a supercomputer research grant, and the Cray C90 at Cray Research Inc. in Eagen, Minnesota under the sponsorship of Frank Kampe. Their support is greatly appreciated.

\section{NOMENCLATURE}

$h \quad$ heat transfer coefficient

$K$ thermal conductivity of coolant

$\mathrm{Nu} \quad$ local Nusselt number, $h D_{h} / K$

$N u_{o} \quad$ Nusselt number in fully-developed turbulent non-rotating tube flow

Pr Prandtl number

Re Reynolds number, $\rho W_{b} D_{h} / \mu$

$T \quad$ local coolant temperature

$T_{o} \quad$ coolant temperature at inlet

$T_{w} \quad$ wall temperature

$W_{b} \quad$ bulk velocity in streamwise direction

$\rho \quad$ density of coolant

$\Delta \rho / \rho$ coolant-to-wall density ratio, $\left(T_{w}-T_{o}\right) / T_{w}$ 
$\theta \quad$ dimensionless temperature, $\left(T-T_{o}\right) /$

$\left(T_{w}-T_{o}\right)$

$\mu \quad$ dynamic viscosity of coolant

\section{References}

Chen, H. C. and Chen, M. (1998) Chimera RANS Simulation of a Berthing DDG-51 Ship in Translational and Rotational Motions, International Journal of Offshore and Polar Engineering, 8(3), 182-191.

Chen, H. C. and Korpus, R. (1993) Multi-block Finite-Analytic Reynolds-Averaged Navier-Stokes Method for 3D Incompressible Flows, ASME Fluids Engineering Conference, FED, 150, $113-121$.

Chen, H. C. and Liu, T. (1999) Turbulent Flow Induced by a Full-Scale Ship in Harbor, Journal of Engineering Mechanics, 125(7), 827-835.

Chen, H. C. and Patel, V. C. (1989) The Flow Around WingBody Junctions, Proceedings of the 4th Symposium on Numerical and Physical Aspects of Aerodynamic Flows, Long Beach, CA

Chen, H. C. (1995a) Assessment of a Reynolds Stress Closure Model for Appendage-Hull Junction Flows, Journal of Fluids Engineering, 117(4), 557-563.

Chen, H. C. (1995b) Submarine Flows Studied by SecondMoment Closure, Journal of Engineering Mechanics, 121(10), $1136-1146$

Chen, H. C., Jang, Y. J. and Han, J. C., Computation of heat transfer in rotating two-pass square channels by a secondmoment closure model, Int. J. Heat and Mass Transfer, 43(9), May, 2000, 1603-1616.

Chen, H. C., Patel, V. C. and Ju, S. (1990) Solutions of Reynolds-Averaged Navier-Stokes Equations for ThreeDimensional Incompressible Flows, Journal of Computational Physics, 88(2), 305-336.

Ekkad, S. V. and Han, J. C. (1997) Detailed Heat Transfer Distributions in Two-Pass Square Channels with Rib Turbulators, Int. J. Heat and Mass Transfer, 40(11), 2525-2537.
Hubbard, B. J. and Chen, H. C. (1994) A Chimera Scheme for Incompressible Viscous Flows with Applications to Submarine Hydrodynamics, AIAA Paper 94-2210, 25th AIAA Fluid Dynamics Conference, Colorado Springs, CO.

Iacovides, H. and Raisee, M. (1999) Recent Progress in the Computation of Flow and Heat Transfer in Internal Cooling Passages of Turbine Blades, International Journal of Heat and Fluid Flow, 20, 320-328.

Iacovides, H. (1998) Computation of Flow and Heat Transfer Through Rotating Ribbed Passage, International Journal of Heat and Fluid Flow, 19, 393-400.

Jang, Y. J., Chen, H. C. and Han, J. C. (2000) Computation of Flow and Heat Transfer in Two-Pass Channels with $60^{\circ}$ Ribs, AIAA Paper 2000-1036.

Kays, W. M. and Crawford, M. E. (1993) Convective Heat and Mass Transfer, Third edition, McGraw Hill.

Liou, T. M., Hwang, J. J. and Chen, S. H. (1993a) Simulation and Measurement of Enhanced Turbulent Heat Transfer in a Channel with Periodic Ribs on one Principal Wall, Int. J. Heat and Mass Transfer, 36, 507-517.

Liou, T. M., Wu, Y. Y. and Chang, Y. (1993b) LDV Measurements of Periodic Fully Developed Main and Secondary Flows in a Channel with Rib-Disturbed Walls, Journal of Fluids Engineering, 115, 109-114.

Prakash, C. and Zerkle, R. (1995) Prediction of Turbulent Flow and Heat Transfer in a Ribbed Rectangular Duct With and Without Rotation, Journal of Turbomachinery, 177, $255-264$.

Rigby, D. L. Steinthorsson, E. and Ameri, A. A. (1997) Numerical Prediction of Heat Transfer in a Channel with Ribs and Bleed, ASME Paper 97-GT-431.

Rigby, D. L. (1998) Prediction of Heat and Mass Transfer in a Rotating Ribbed Coolant Passage with a 180 Degree Turn, ASME Paper 98-GT-329.

Stephens, M. A., Chyu, M. K. and Shih, T. I.-P. (1996) Computation of Convective Heat Transfer in a Square Duct with Inclined Ribs of Rounded Cross Section, ASME Paper 96-WA/HT-12.

Stephens, M. A., Shih, T. I.-P. and Civinskas, K. C. (1995) Computation of Flow Heat Transfer in a Rectangular Channel with Ribs, AIAA Paper 95-0180. 

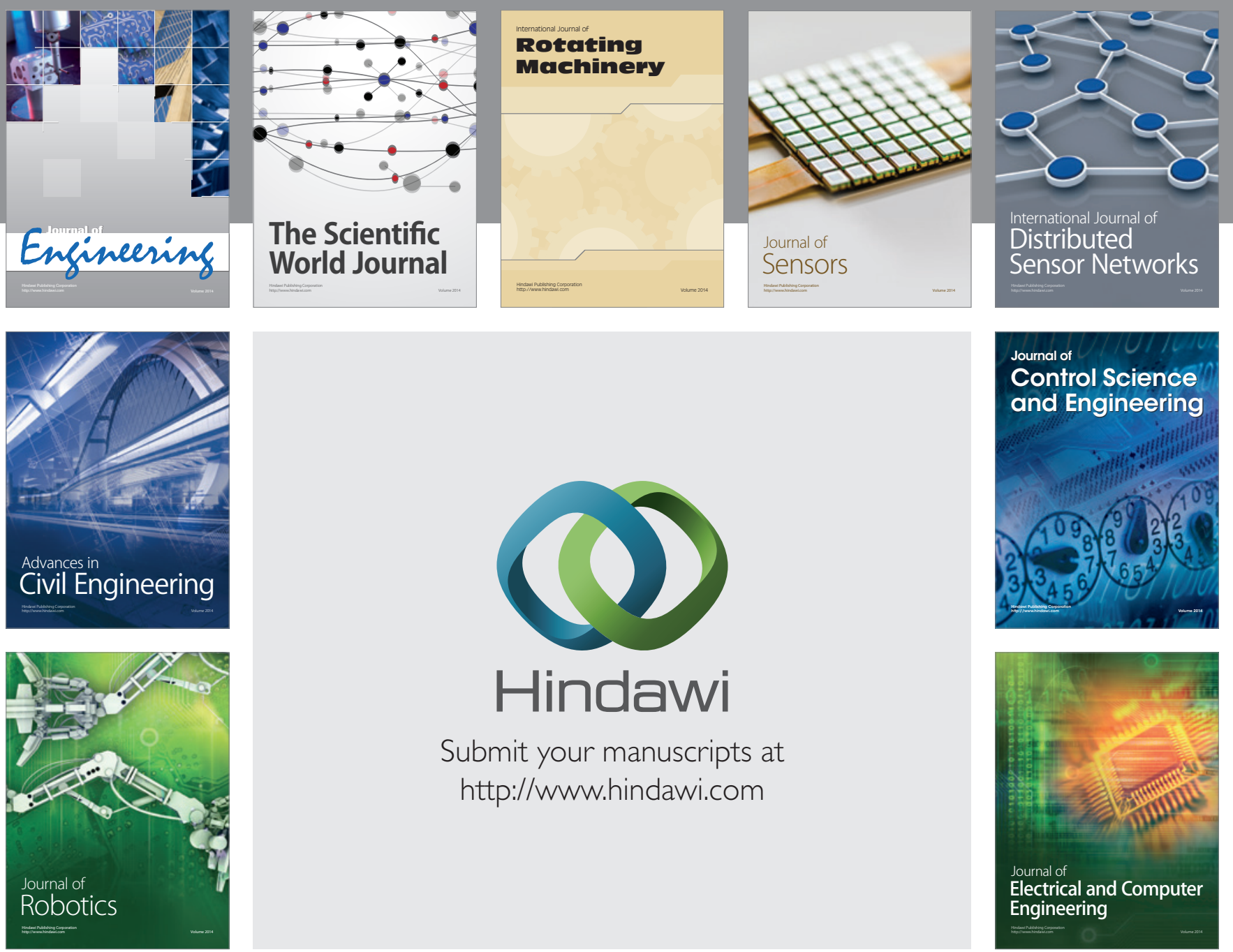

Submit your manuscripts at

http://www.hindawi.com
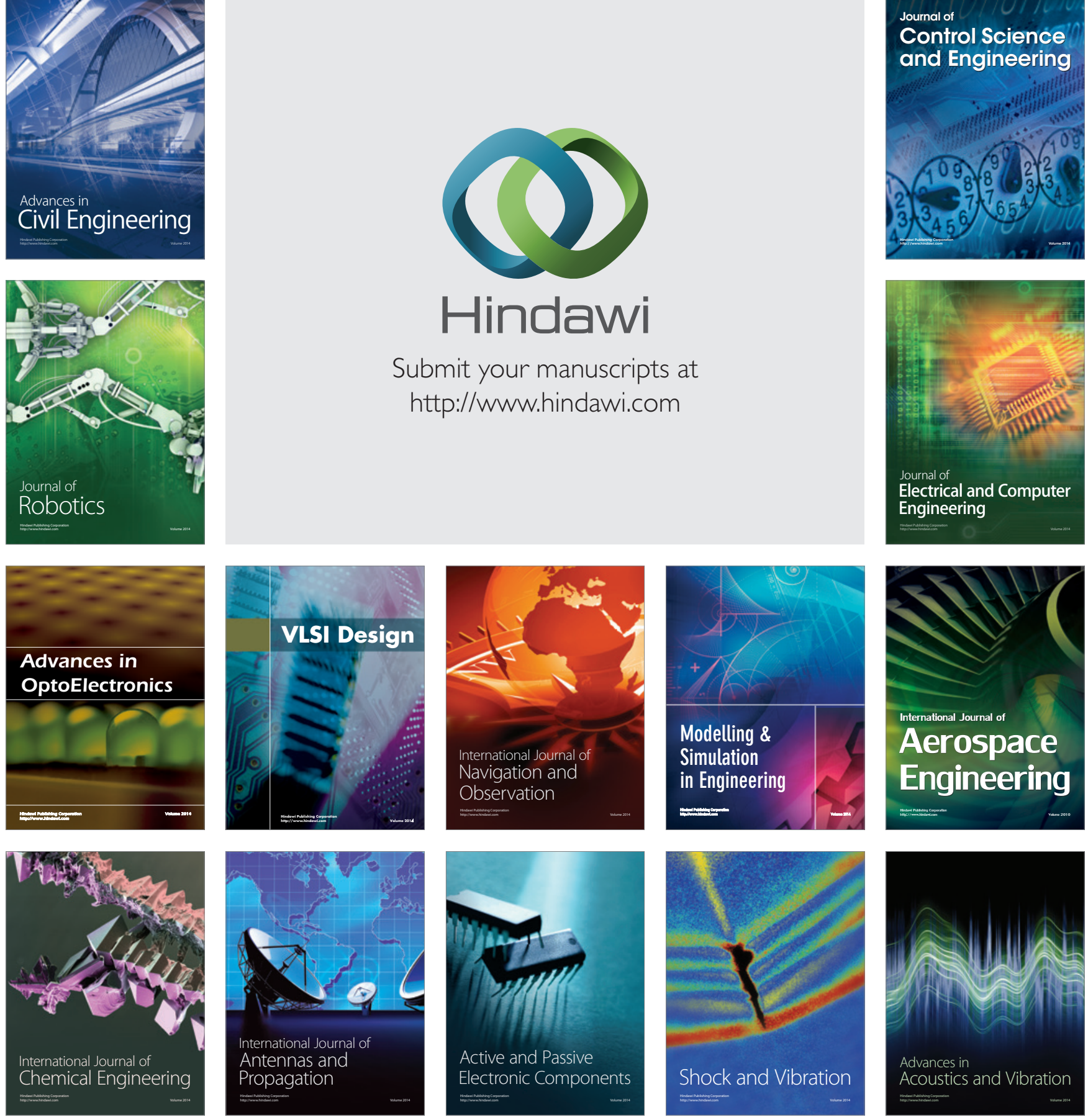